\title{
Zero mode topology with dynamical overlap fermions
}

\section{Nigel Cundy*}

Theoretische Physik, Universität Wuppertal, Gaussstrasse 19, 42119 Wuppertal, Germany.

Email: cundyatheorie.physik.uni-wuppertal.de

\section{Stefan Krieg, Thomas Lippert,}

Central Institute for Applied Mathematics, Forschungszentrum Jülich GmbH, D-52425 Jülich,

Germany

\begin{abstract}
We investigate the correlation between the topological charge density and the small eigenvectors of the overlap Dirac operator on ensembles generated at four different quark masses using dynamical overlap fermions. We identify topological structures in the QCD vacuum, and by investigating their size, shape and topological charge, we determine that the small eigenvalues of the Dirac operator are dominated by approximately hyper-spherical objects with integer topological charge.
\end{abstract}

XXIVth International Symposium on Lattice Field Theory

July 23-28, 2006

Tucson, Arizona, USA

${ }^{*}$ Speaker. 


\section{Introduction}

An open question in lattice QCD is the cause of the spontaneous breaking of the $S U\left(N_{f}\right) \otimes$ $S U\left(N_{f}\right)$ symmetry in the massless QCD Lagrangian. The order parameter of this chiral symmetry breaking is the chiral condensate, proportional to the density of zero eigenvalues of the Dirac operator. It is likely that the near-zero eigenvectors are generated by the mixing of "would be zero modes," topological objects with non-zero winding number. In Euclidean space time, many models of the topological vacuum have been suggested, including: point-like and hyper-spherical instantons with integer topological charge, monopoles or calorons with fractional charge, vortexes, higher dimensional membranes or perhaps some combination of the above. In principle, we can eliminate some of these possibilities by searching for anti-self dual objects in the Dirac operator, and studying their size, shape, and correlation with the topological charge density. In practise, there are numerous difficulties, caused by both identification of the structures, from finite lattice spacing effects, and from ultra-violet fluctuations.

We have to use a Dirac operator which respects chiral symmetry, such as the overlap operator [1]:

$$
D=(1+\mu)+(1-\mu) \gamma_{5} \operatorname{sign}\left(\gamma_{5} D_{W}\right)
$$

where $D_{W}$ is the Wilson Dirac operator with negative mass (we use a hopping parameter $\kappa=0.2$ ), and $\mu$ is proportional to the bare quark mass. Studies in quenched QCD have not yet produced conclusive results. In the most recent studies, Horvath et al. believe that they have identified long range three dimensional membranes in the underlying gauge field, [2]; while Weinberg et al. have found a different picture emerging if one only considers the lattice sites with the largest topological charge density [3]; and Wang et al. [4] believe that the vacuum is dominated by instantons, with Horvath incorrectly accounting for ultra-violet fluctuations. However, since the fermion determinant (at small masses) will suppress small eigenvalues of the Dirac operator, it is likely that dynamical effects will be important.

This study is a preliminary investigation of the topological structure of the QCD vacuum in full QCD (with overlap fermions). We are currently restricted to small lattices, and large quark masses because of the costs of the Dynamical overlap simulations. In the quenched studies it was found that finite lattice spacing effects were important; we are not yet ready to confirm this in full QCD: this is the most important limitation of our work as presented here.

In section 2 we describe the parameters used for our ensembles. In section 3 we describe our method. In section 4 we give our results, and we conclude in section 5 .

\section{Setup}

We generated our ensembles using the Hybrid Monte Carlo algorithm for overlap fermions described in $[5,6,7]$. We generated five $8^{3} 32$ ensembles, each with around 20 configurations, at a lattice spacing of either $0.15 \mathrm{fm}$ or $0.1 \mathrm{fm}$ at pion masses ranging from $400 \mathrm{MeV}$ to $1200 \mathrm{MeV}$ (see table 1). We used the Tadpole-Improved Lüscher Weisz gauge action and two steps of stout smearing at a parameter 0.1. Note that the ensembles at the two lowest masses had few small eigenvalues of either the overlap or Wilson operator or many topological charge changes. It is likely that these two masses were too small to fit into the physical volume. 


\begin{tabular}{llllllll} 
Size & $\beta$ & $\mathrm{a}(\mathrm{fm})$ & $\mu$ & $m_{\pi}(\mathrm{MeV})$ & $\operatorname{Re}\left(P_{l}\right)$ & $\operatorname{Im}\left(P_{l}\right)$ & $\left\langle\operatorname{Re}\left(\operatorname{Tr} U_{\mu v}\right) / 3\right\rangle$ \\
\hline $8^{3} 32$ & 8.35 & $0.143(5)$ & 0.04 & $1166(150)$ & $0.008(6)$ & $0.004(12)$ & $0.375(1)$ \\
$8^{3} 32$ & 8.35 & $0.162(7)$ & 0.03 & $852(220)$ & $-0.016(10)$ & $-0.007(10)$ & $0.379(1)$ \\
$8^{3} 32$ & 8.45 & $0.101(7)$ & 0.01 & $617(200)$ & $0.037(15)$ & $-0.011(7)$ & $0.363(1)$ \\
$8^{3} 32$ & 8.45 & $0.109(15)$ & 0.008 & $414(300)$ & $0.029(8)$ & $-0.024(19)$ & $0.363(1)$
\end{tabular}

Table 1: Ensemble details, the size of the lattice, $\beta$, quark mass, pion mass, Real and imaginary values of the Polyakov loop, and average plaquette.

\section{Method}

We calculate the eigenvectors $\left|\psi_{i}\right\rangle$ (with eigenvalue $\lambda_{i}$ closest to 0 ) of the massless Hermitian overlap operator. We define a scalar density $\psi_{i}(x)$ and pseudo-scalar density $\psi_{i}^{5}(x)$

$$
\psi_{i}(x)=\sum_{c, s}\left\langle\psi_{i}(x) \mid \psi_{i}(x)\right\rangle \quad \psi_{i}^{5}(x)=\sum_{c, s}\left\langle\psi_{i}(x)\left|\gamma_{5}\right| \psi_{i}(x)\right\rangle .
$$

We use a topological charge density based on a construction of $F_{\mu \nu}$ using $1 \times 1,2 \times 2$ and $3 \times 3$ Wilson loops $[8,9]$. This gives an $\mathrm{O}\left(a^{4}\right)$ improved topological charge density, with the UV fluctuations removed by an improved cooling method so that the total topological charge is an integer to better than $1 \%$. We compare the eigenvectors with the topological charge density calculated after $5,10,15, \ldots 40$ cooling sweeps, with the hope that this will allow us to determine which structures we see are artifacts of the cooling and which were in the original gauge field.

So that we can determine the nature of the underlying structures or objects in the eigenvectors, we must first isolate those structures so that we can investigate their shape, size and topological charge. Finding connected regions with coherent chirality is not enough - this gives us two structures covering all of the lattice (the two long range structures found by Horvath $e t a l$ ). We can attempt to insert a cut-off, only considering a certain fraction of the lattice sites with the highest (pseudo-)scalar density, but again this is not sufficient to separate these large structures into smaller objects without excluding a large proportion of the lattice. We can, however, split the (pseudo-)scalar density and topological charge into several different structures based on the gradient of $\psi_{i}, \psi_{i}^{5}$ or $q_{g}$. The goal is to assign each lattice site to one particular structure. The following procedure is for $\psi_{i}$, with the method for $\psi_{i}^{5}$ or $q_{g}$ being analogous.

We start by identifying the lattice site with the highest $\psi_{i}$, and we assign this lattice site to the first structure, together with its nearest neighbours. Then for each lattice site $x_{n}$ adjacent to the structure, we calculate the gradient of $\psi_{i}$ from the $3^{4}$ hypercube centred on $x_{n}$. We map this gradient vector onto the $3^{4}$ hypercube (by starting the vecor at $x_{n}$ and multiplying by an appropriate scale factor), and find the two lattice sites on the hypercube nearest to the mapped gradient vector. If either of these sites have already been assigned to the first structure, then we assign $x_{n}$ to the same structure. If not, we leave it unassigned for the present time. We continue to repeatedly sweep through the lattice until no more lattice sites can be assigned to the this structure. We can take the lattice site with the highest $\psi_{i}$ which is not assigned to the first structure as a starting point for the second structure, and repeat this procedure until no more lattice sites are left unassigned. This procedure generates numerous connected structures scattered across the lattice. It has its flaws, most notably that it may be biased towards hyper-spherical structures (a one dimensional ridge, for 
example, may be split between several structures); secondly, it can break down if $\psi_{i}$ is insufficiently smooth. None the less, this method, though crude, is still effective, as we hope to demonstrate later.

Once we have individual structures, we need to determine their size, shape, topological charge and the correlation between the object and the topological charge. To study their size and shape, we here use a somewhat primitive method - we plot the scalar/pseudo-scalar/topological charge densities and examine them by eye. We are currently developing more sophisticated tools.

We also construct straight line paths across the structures. For every two lattice sites within the structure we can construct at least one path connecting these two sites (where the path is not allowed to go outside the structure) which minimises the length of the path. Then, of all the possible minimum-length paths in the structure we choose the one path which has the largest scalar density (or absolute value of the pseudo-scalar density) on the lattice sites along that path. This gives us a one-dimensional view across the most important lattice sites in the structure; and we can compare the pseudo-scalar density and topological charge density along this path, most simply by plotting them together. We can also determine information about the shape of the structure from the path. Because we are choosing the path of maximum scalar density, then if the structure is in the form of one or higher dimensional object, of (almost) constant scalar density, then this should be evident, at least on some configurations, as we will either see a ridge of constant pseudo-scalar density, or (at least) two peaks as the path crosses the structure twice. If there are at least two hyper-spherical peaks, then we should see both of them (at least most of the time). Only if the structures are generated by one hyper-spherical object would we universally see a single peak in the path.

To calculate the topological charge of the structures, we find a structure in the topological charge density with its maximum located near (we chose to use those within $\sqrt{6}$ lattice spacings and only two lattice spacings distant in one direction) a maximum in the (pseudo-)scalar density structure, then summing up $q_{g}$ over the lattice sites assigned to the topological charge structure. For the larger objects which we are interested in here, the topological charge generated by summing $q_{g}$ over the lattice sites in the (pseudo-)scalar density structures was almost invariably close to that obtained from the corresponding topological charge structure. In this study, since we were interested in the topological content of the eigenvectors, we only considered those structures which appeared in all of $\psi, \psi^{5}$ and $q_{g}$.

\section{Results}

In figure 1 we plot the pseudo-scalar density for the eigenvector with the smallest eigenvalue in the $\mu=0.03$ ensemble. We see that the pseudo-scalar density is dominated by two structures: one with positive $\psi^{5}$, the other with negative $\psi^{5}$; and that both of these objects are approximately hyper-spherical (in the sense that around the peak the pseudo-scalar density falls off at the same rate in every direction): we certainly do not see $3 \mathrm{D}$ membranes of roughly constant density. We can separate the two structures using the gradient method, and we have plotted the pseudo-scalar density of one of them in 2 (the other is similar). After 5 cooling sweeps the topological charges of these two objects were -0.937 and 0.851 ; after 10 cooling sweeps -1.019 and 0.958 . We can confirm that we are seeing the same structures in both the topological charge density and pseudoscalar density by plotting $q_{g}$ and $\psi^{5}$ along the path of maximum $\left|\psi^{5}\right|$ (see figure 3 ). While the gauge field is not very smooth, since it was calculated after only 5 cooling sweeps, it is clear that 


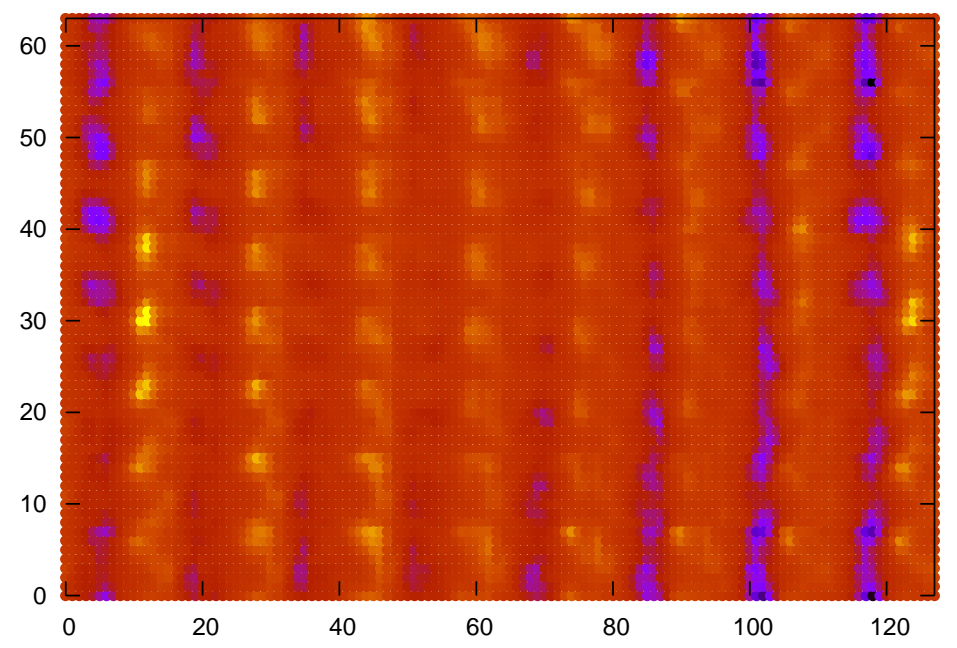

Figure 1: The distribution of $\psi^{5}$ on the lattice for the smallest eigenvalue of the $\mu=0.03$ ensemble. The $\mathrm{X}$-axis contains the $\mathrm{X}$ and $\mathrm{T}$ coordinates of the original lattice $\left(x=L_{X} T+X\right)$; the $\mathrm{y}$-axis the $\mathrm{Y}$ and $\mathrm{Z}$. A dark colour indicates a large negative value, a yellow colour a large positive value, and a red colour a value around zero.

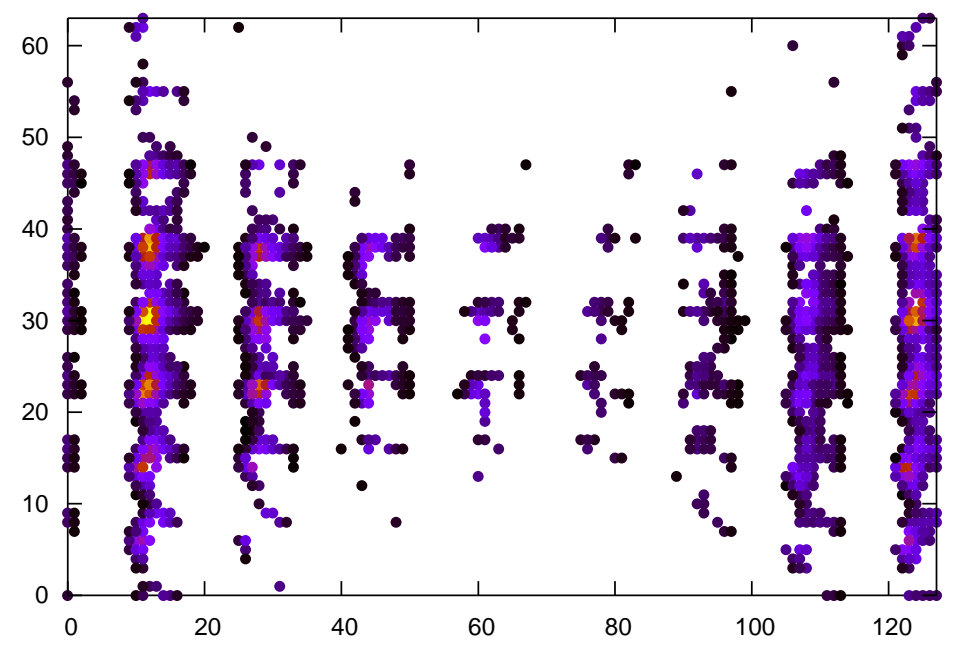

Figure 2: The same plot as in figure 1, but restricted to just the structure with the largest topological charge. A light colour indicates a large value of $\psi^{5}$, a dark colour a value close to zero. 


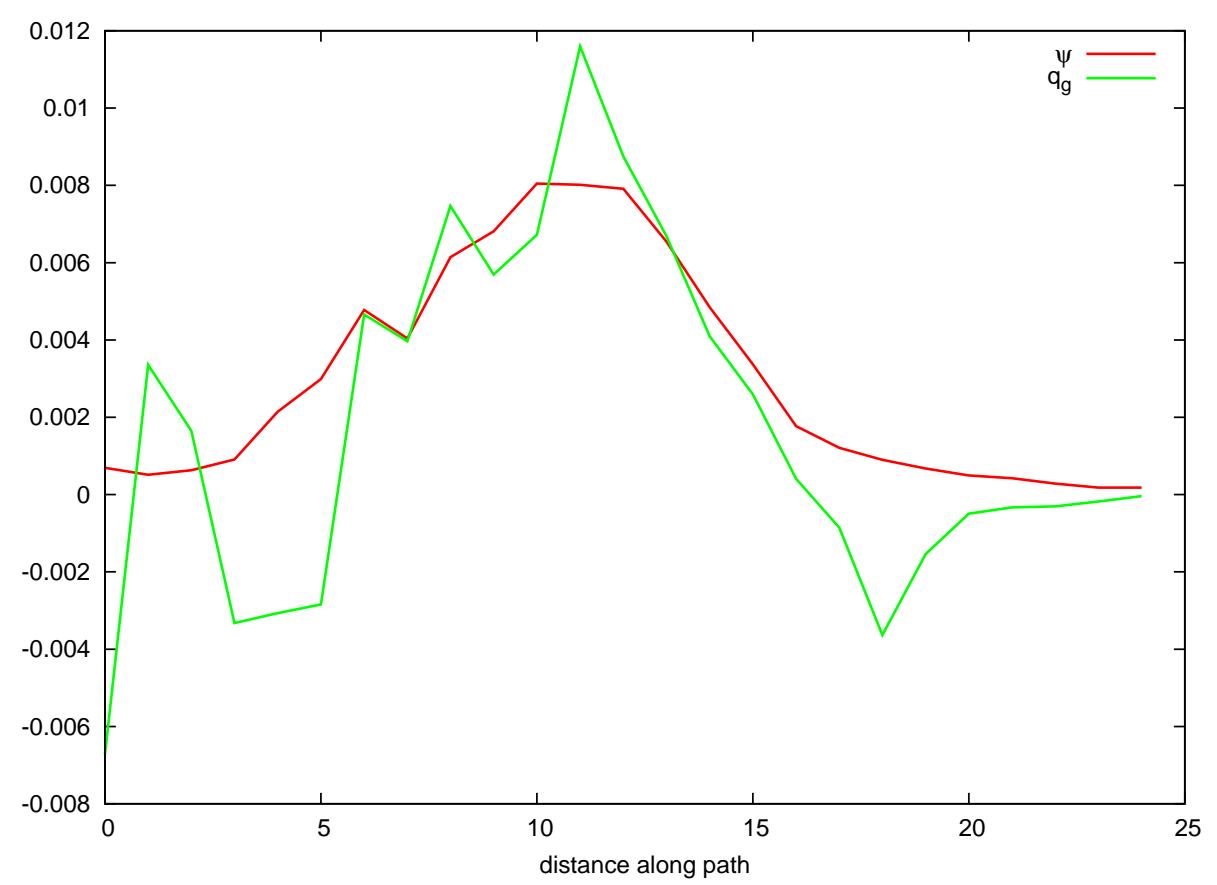

Figure 3: The pseudo-scalar density for the smallest eigenvector of the $\mu=0.03$ ensemble and the topological charge density (calculated after 5 cooling sweeps) along the path with the maximum $\left|\psi_{5}\right| . q_{g}$ has been scaled by a factor of 10 .

the same structure is seen in both the gauge field and the eigenvector. Since the eigenvector was calculated on the uncooled gauge field, it is clear that this structure is not an artifact of cooling. The third and fourth structures in $\psi^{5}$ both had topological charges around - 0.4 (if we summed up the charges of the sites in the pseudo-scalar density structures), neither of which was well correlated to the topological charge density; indeed a structure in $q_{g}$ with charge -1.2 was split across these two eigenvector structures (and others). It thus seems highly likely that this small eigenvector of the Dirac operator was created by the mixing of two hyper-spherical objects, one of charge +1 , the other of charge -1 .

That was one eigenvector on one configuration on one ensemble. Now we just have to perform the rather tedious task of repeating this procedure for the remaining eigenvectors, configurations, and ensembles. We see four distinct classes of eigenvectors. There are class (a) eigenvectors which contain (at least) two structures of topological charge one, as in the example discussed above (all structures with charges between 0.8 and 1.2 were determined to be charge 1 ; these structures were generally stable under cooling). Class (b) eigenvectors had one topological charge 1 structure and a number of structures with topological charges around 0.5. Class (c) eigenvectors had a single topological charge one structure, and class (d) eigenvectors had no obvious correlation with the topological charge (according to this method). Our results are summarised in table 2 for the smallest non-zero eigenvectors for each configurations. On the $\mu=0.03$ and $\mu=0.04$ ensembles the eigenvectors are mostly class (a), with the occasional class (b) or (c). For the smaller masses, where we had no small eigenvalues, we saw only class (c) and class (d) eigenvectors. The number of class (a) eigenvectors on the $\mu=0.03$ and $\mu=0.04$ ensembles decreased as we moved to 


\begin{tabular}{lllll}
$\mu$ & (a) & (b) & (c) & (d) \\
\hline 0.04 & $70 \%$ & $20 \%$ & $10 \%$ & $0 \%$ \\
0.03 & $60 \%$ & $25 \%$ & $15 \%$ & $0 \%$ \\
0.01 & $10 \%$ & $0 \%$ & $30 \%$ & $60 \%$ \\
0.008 & $0 \%$ & $0 \%$ & $50 \%$ & $50 \%$
\end{tabular}

Table 2: The percentage of configurations where the smallest non-zero eigenvector (counting only structures seen in $\psi, \psi^{5}$ and $q_{g}$ ) belonged to one of the four classes defined in the text.

higher eigenvalues - there was generally considerably less correlation between structures in the eigenvector and the topological charge.

\section{Conclusions}

In contrast to the quenched study of [2] we find that the small eigenvalues of the Dirac operator are dominated by hyper-spherical structures with topological charge of around 1 . This discrepancy could be caused by a number of factors; firstly the presence of dynamical fermions; secondly that our study was carried out on smaller volumes and with a larger lattice spacing; thirdly because it is possible that our cooling method of measuring the topological charge introduces a bias into the method. Further study is needed to investigate which of these is the cause of the discrepancy. Our results are not inconsistent with a picture in which the dominant cause of spontaneous chiral symmetry breaking is some remnant of instanton/anti-instanton pairs.

\section{References}

[1] H. Neuberger, Phys. Rev. Lett. 81, 4060 (1998) [arXiv:hep-lat/9806025].

[2] I. Horvath et al., Phys. Lett. B 61221 (2005) [arXiv:hep-lat/0501025].

[3] E. M. Ilgenfritz, K. Koller, Y. Koma, G. Schierholz, T. Streuer and V. Weinberg, Nucl. Phys. Proc. Suppl. 153328 (2006) [arXiv:hep-lat/0512005].

[4] Z. Q. Wang, X. F. Lu and F. Wang, arXiv:hep-lat/0606015.

[5] N. Cundy, Nucl. Phys. Proc. Suppl. 15354 (2006) [arXiv:hep-lat/0511047].

[6] N. Cundy, S. Krieg, G. Arnold, A. Frommer, T. Lippert and K. Schilling, arXiv:hep-lat/0502007.

[7] N. Cundy, S. Krieg and T. Lippert, PoS LAT2005 107 (2006) [arXiv:hep-lat/0511044].

[8] J. B. Zhang, S. O. Bilson-Thompson, F. D. R. Bonnet, D. B. Leinweber, A. G. Williams and J. M. Zanotti, Phys. Rev. D 65074510 (2002) [arXiv:hep-lat/0111060].

[9] S. Bilson-Thompson, F. D. R. Bonnet, D. B. Leinweber and A. G. Williams, Nucl. Phys. Proc. Suppl. 116 109A (2002) [arXiv:hep-lat/0112034]. 